OPEN ACCESS

Edited by:

Linda F. Van Dyk,

University of Colorado Denver,

United States

Reviewed by:

Christopher J. A. Duncan, Newcastle University, United Kingdom

Chunfu Zheng,

Fujian Medical University, China Martin R. Jakobsen,

Aarhus University, Denmark

${ }^{*}$ Correspondence:

Richard B. Kennedy

kennedy.rick@mayo.edu

Specialty section:

This article was submitted to

Viral Immunology,

a section of the journal

Frontiers in Immunology

Received: 29 May 2020

Accepted: 24 September 2020

Published: 14 October 2020

Citation:

Kennedy RB, Haralambieva IH,

Ovsyannikova IG, Voigt EA,

Larrabee BR, Schaid DJ,

Zimmermann MT, Oberg AL and Poland GA (2020) Polymorphisms in STING Affect Human Innate Immune Responses to Poxviruses.

Front. Immunol. 11:567348. doi: 10.3389/fimmu.2020.567348

\section{Polymorphisms in STING Affect Human Innate Immune Responses to Poxviruses}

\author{
Richard B. Kennedy ${ }^{1 *}$, lana H. Haralambieva ${ }^{1}$, Inna G. Ovsyannikova ${ }^{1}$, Emily A. Voigt ${ }^{1}$, \\ Beth R. Larrabee ${ }^{2}$, Daniel J. Schaid ${ }^{2}$, Michael T. Zimmermann ${ }^{3}$, Ann L. Oberg ${ }^{2}$ \\ and Gregory A. Poland ${ }^{1}$
}

\begin{abstract}
${ }^{1}$ Mayo Clinic Vaccine Research Group, Mayo Clinic, Rochester, MN, United States, ${ }^{2}$ Division of Biomedical Statistics and Informatics, Department of Health Science Research, Mayo Clinic, Rochester, MN, United States, ${ }^{3}$ Bioinformatics Research and Development Laboratory, Genomics Sciences and Precision Medicine Center, Medical College of Wisconsin, Milwaukee, WI, United States
\end{abstract}

We conducted a large genome-wide association study (GWAS) of the immune responses to primary smallpox vaccination in a combined cohort of 1,653 subjects. We did not observe any polymorphisms associated with standard vaccine response outcomes (e.g., neutralizing antibody, T cell ELISPOT response, or T cell cytokine production); however, we did identify a cluster of SNPs on chromosome $5(5 \mathrm{q} 31.2)$ that were significantly associated ( $\mathrm{p}$-value: $1.3 \times 10^{-12}-1.5 \times 10^{-36}$ ) with IFN $\alpha$ response to in vitro poxvirus stimulation. Examination of these SNPs led to the functional testing of rs1131769, a nonsynonymous SNP in TMEM173 causing an Arg-to-His change at position 232 in the STING protein - a major regulator of innate immune responses to viral infections. Our findings demonstrate differences in the ability of the two STING variants to phosphorylate the downstream intermediates TBK1 and IRF3 in response to multiple STING ligands. Further downstream in the STING pathway, we observed significantly reduced expression of type I IFNs (including IFN $\alpha$ ) and IFN-response genes in cells carrying the $\mathrm{H} 232$ variant. Subsequent molecular modeling of both alleles predicted altered ligand binding characteristics between the two variants, providing a potential mechanism underlying differences in inter-individual responses to poxvirus infection. Our data indicate that possession of the H232 variant may impair STING-mediated innate immunity to poxviruses. These results clarify prior studies evaluating functional effects of genetic variants in TMEM173 and provide novel data regarding genetic control of poxvirus immunity.

Keywords: STING, smallpox vaccine, vaccinia, poxvirus, viral vaccines, immunogenetics, innate immunity, genomewide association study 


\section{INTRODUCTION}

Until its eradication in 1980, smallpox (caused by the variola virus) was a deadly, debilitating disease estimated to have killed hundreds of millions of individuals over the last two centuries alone (1). Eradication was made possible by using vaccines based on vaccinia virus (1). These live-virus vaccines elicited robust, long-lasting immunity in nearly all vaccine recipients $(2,3)$. Routine smallpox vaccination was halted in the United States before global eradication due to rare but serious adverse events, including death; however, poxviruses remain a public health issue for several reasons, including zoonotic poxvirus outbreaks (4-7) and concerns regarding the release of variola virus as a biological weapon and novel poxviruses (8). The increasing use of poxviruses as platform vectors for other vaccines and therapeutics has also enhanced our need for a better understanding of poxvirus immunity. While highly effective, the smallpox vaccine has numerous contraindications as well as rare but serious, potentially life-threatening adverse reactions that limit its widespread use, if needed, in the population. Understanding how poxvirus immunity is controlled may assist in the development of safer yet still effective poxvirusbased vaccines and can provide insights into immunity to other DNA viruses/vaccines.

Although seroconversion rates after smallpox vaccination are high (>97\%), antibody titers and cellular immune responses vary widely among recipients $(1,9-12)$. We have previously reported on a small subset of individuals who develop the classical vaccine take (i.e., response) but fail to mount vigorous adaptive immune responses (13). Previous research by our lab and others demonstrates that genetic polymorphisms are correlated with immune outcomes to multiple viral vaccines, including the smallpox vaccine $(11,12,14,15)$. To move beyond statistical genetic associations, functional studies are also needed to elucidate the biologic mechanisms underlying these associations and link them to gain a better understanding of how genomic factors contribute to inter-individual variation in immune response.

Recognition of foreign nucleic acids by the cGAS/STING pathway leads to type I IFN responses and is an important component of the innate response to viral and bacterial infection (16). Cytosolic DNA is recognized by cGAS, leading to the generation of the cyclic dinucleotide 2'3'cGAMP, which, in turn binds to STING. STING is also able to directly recognize bacterially produced cyclic dinucleotides. Both direct activation of STING and indirect activation through cGAS trigger the phosphorylation of TBK1 and IRF3, resulting in the transcription of type I interferons, TNF, IL-6, with subsequent activation of interferon regulated genes and inflammation. STING has been found to mediate the IFN response to bacteria and DNA viruses, including herpes simplex viruses, $\mathrm{CMV}, \mathrm{HPV}$, and poxviruses such as vaccinia and ectromelia (17-21).

We have previously reported the findings from the first genome-wide association study (GWAS) examining the association of single nucleotide polymorphisms (SNPs) with immune responses in a cohort of primary smallpox vaccines
$(11,12,15)$. Here, we report a cluster of SNPs on chromosome 5 (5q31.2) that were significantly associated with IFN $\alpha$ response following in vitro stimulation of PBMCs with vaccinia virus. We report the results from the functional testing of rs1131769, which is a non-synonymous SNP in TMEM173 that introduces an amino acid change from the arginine at position 232 (R232) to histidine (H232) in the STING protein (18-21). Our results indicate that the $\mathrm{H} 232$ variant of STING is associated with a significant reduction in the IFNa response and that this effect is independent of the effect previously described for SNPs in the STING HAQ haplotype (22). We also report the results of molecular modeling and molecular dynamics (MD) simulations investigating differences in how the $\mathrm{H} 232$ and R232 variants interact with the signaling ligand. Overall, our study provides novel and important data regarding genetic control of poxvirus immunity in humans by linking specific genetic polymorphisms in TMEM173 to differential STING pathway activation during innate immune responses (IFN $\alpha$ ) to vaccinia virus. These results may also explain inter-individual variations in the innate immune response to other DNA viruses (e.g., HPV, VZV, HSV-1), which also stimulate the STING pathway, as well as the large number of bacterial pathogens that also activate STING.

\section{MATERIALS AND METHODS}

\section{Study Cohorts}

Two previously described study cohorts were combined for our analyses $(11,12,15)$. Briefly, the San Diego cohort consists of 1,076 Dryvax ${ }^{\circledR}$ recipients (primarily U.S. Navy personnel) recruited in 2003-2006. The U.S. cohort consists of 1,058 ACAM $2000^{\circledR}$ or Dryvax ${ }^{\circledR}$ recipients (primarily U.S. Army personnel) recruited in 2010-2013. For both cohorts, subjects had received their first (and only) smallpox vaccination between 1 and 4 years prior to study enrollment. All participants gave written informed consent for this study. Approval for all study procedures was obtained from the Institutional Review Boards of the Mayo Clinic (Rochester, MN) and the Naval Health Research Center (NHRC; San Diego, CA).

\section{Measurement of Vaccinia-Specific IFN $\alpha$ Responses}

Subject PBMC samples were cultured in the presence/absence of inactivated vaccinia virus $(\mathrm{NYCBOH})$ at an $\mathrm{MOI}$ of 0.05 . Vaccinia virus was inactivated using psoralen $(5 \mathrm{ug} / \mathrm{ml}$ : Sigma Aldrich, P8399) and long-wave UV light (23). The full panel of cytokines included: IL-1 $\beta$, IL-2, IL-4, IL-6, IL-10, IL-12p40, IL12 p70, IL-18, IFN $\alpha$, IFN $\beta$, and TNF $\alpha$. Interferon alpha (IFN $\alpha$ ) production by vaccinia virus-stimulated PBMC samples was measured by commercial ELISA assay (PBL Biomedical Laboratories, Piscataway, NJ), as previously described $(15,24)$. PHA was used as a viability control. Cells from subjects that were unable to secrete cytokines (e.g., IFN $\gamma, \mathrm{TNF} \alpha$, IL-2) upon stimulation by PHA were deemed unviable and not included in the analyses. 


\section{Genotyping and Fine Mapping}

DNA from all subjects was extracted using Gentra Puregene kits (QIAGEN, \#158445) (11). Genome-wide genotyping for the study cohort was performed as previously described $(11,12$, 15, 25). For the SD cohort (recruited in 2003-2006), subjects were genotyped with either the Illumina 550 array or the Illumina 650 array. Genotype quality control (QC) prior to imputation was conducted separately for each platform. QC for the Illumina 550 and 650 arrays involved removing monomorphic SNPs and those on the Y chromosome. We also removed all SNPs with a call rate $<95 \%$, and all subjects with a call rate $<95 \%$. SNPs were also excluded if they failed HardyWeinberg Equilibration (HWE) test p-value $>10^{-5}$. Genetic sex was verified by PLINK. Subjects in the U.S. cohort were recruited in 2010-2013 and genotyped with the Illumina Omni 2.5 array. For the Omni 2.5 array, mitochondrial SNPs, those on the Y chromosome, and monomorphic SNPs were removed. SNPs were required to have a call rate at least $99 \%$, and subjects had a minimum call rate of $95 \%$. No inconsistencies were found between reported sex and genetic-determined sex. Across these cohorts, a total of 2,062 subjects passed QC for genotyping.

The 1000 Genomes cosmopolitan samples (Build 37: African, AFR; American, AMR; Asian, ASN; European, EUR) served as a reference for SNP imputation. Observed SNPs were eliminated prior to imputation if they could not be converted to the forward strand or if more than one SNP mapped to a given position. The reference genome was filtered to exclude SNPs whose minor allele frequency (MAF) was $<0.005$. The data were then phased using SHAPEIT (26) and imputed via IMPUTE2 (27). SNPs were included in analyses if their imputation dosage allele $\mathrm{R}^{2}$ was at least 0.3 and their MAF was at least 0.01 . These GWAS QC restrictions resulted in a dataset with 6,210,296 SNPs for the HumHap550 array; 6,244,529 SNPs for the HumHap650 array; and 6,243,494 SNPs for the Omni 2.5 array.

Fine mapping on the chromosome 5 region was performed using a custom Illumina iSelect panel that included known SNPs in the following gene regions: TMEM173, KCNN2, DNAJC18, and TRIM36 (the coding region, the intronic regions, and $10 \mathrm{~kb}$ upstream and downstream in order to capture regulatory regions). We then identified all SNPs highly correlated ( $\mathrm{r} 2>$ 0.9 ) with each of the target SNPs of interest based on the GWAS results. SNPs were excluded from the fine-mapping effort for the following reasons: low rank on the Illumina design score metric (indicating a low likelihood of successful genotyping); any Illumina error codes; previously genotyped SNPs; and monomorphic SNPs (based on HapMap and 1000 Genomes data). The resulting list of 2,406 SNPs were included on the Illumina iSelect panel. The genotyping was performed in Mayo Clinic's Clinical Genome Facility on 2,208 subjects: 2,011 subjects from the SD and U.S. cohorts; and 197 subjects used for quality control (55 negative controls, 48 trios of father/ mother/child). 1,996 of these subjects passed all QC metrics filters (e.g., call rate at least 99\%, duplicates removed, etc.). Of the genotyped SNPs, a total of 580 SNPs were used in the analysis (156 SNPs failed genotyping; 10 SNPs had call rates < 95\%; 32 SNPs had HWE p-values $<10 \mathrm{E}^{-6}$, and 1,500 were monomorphic). For the Caucasian subgroup, an additional 126 SNPs were removed because they were monomorphic in that subgroup.

\section{Genetic Ancestry and Population Stratification}

Genotypes from the GWAS arrays were used to assign ancestry groups (i.e., Caucasian, African American, or Asian) to participants using the STRUCTURE software (28) and the 1000 Genomes reference data. Genetic ancestry proportions were estimated within cohorts and arrays (San Diego/550, San Diego/650, US/Omni 2.5), as previously described $(11,15)$. A linkage disequilibrium (LD) pruning process (29) was utilized to ensure that the SNPs used for STRUCTURE and for sample eigenvectors were not drawn from small clusters within specific locations (30). Resulting SNPs were entered into to the STRUCTURE program (28), and participant ancestry was classified based on the largest ancestry proportion estimated by STRUCTURE.

Within ancestry groups, eigenvectors were estimated for population-stratification purposes. SNPs with a MAF $<0.01$ and those with a HWE p-value $<0.001$ were excluded, as were insertion/deletions (INDELS). The remaining SNPs were pruned according to the following variance inflation factors: window size of 50 kilobases; step size of 5; and variance inflation factor threshold of 1.05. SmartPCA was used to create the eigenvectors (31) following the procedures implemented in EIGENSTRAT software. Eigenvectors were included as potential covariates if they had a Tracy-Widom p-value $<0.05$.

\section{Selection of Covariates to Adjust for Potential Confounders}

For analysis purposes, the immune-response trait of interest (IFNo secretion) was calculated by first computing the difference of the mean stimulated and unstimulated values and then transforming to a normal distribution using normal quantiles. In order to combine data from the two cohorts, potential confounder effects for each ancestry group and cohort were adjusted by linear regression models as described (29). Categorical variables with a very large number of categories were binned using hierarchical clustering. This was achieved by using hierarchical clustering on the estimated regression coefficients for the different categories while binning categories with similar regression coefficients. Categorical variables were included in regression models by using indicator variables for categories, treating the most common category as baseline. Residuals from the linear models were used as the primary adjusted traits for GWAS analyses.

\section{GWAS Analysis Strategy}

In order to maximize the power to detect SNPs associated with smallpox vaccine immune response phenotypes, data across genotyping arrays and the two cohorts was pooled after preparing the data as described above. The pooled analyses were then performed using the adjusted traits described above in a regression analysis, along with an indicator of cohort as an 
additional adjusting covariate. Because the largest ancestry group was Caucasian, we restricted our pooled analysis to the Caucasian subjects $(n=1,605)$. Multiple testing was controlled for by using the standard $\mathrm{p}$-value threshold ( $\mathrm{p}$-value $<5 \times 10^{-8}$ ) to determine genome-wide statistical significance $(32,33)$. Statistical analyses were performed with the $\mathrm{R}$ statistical software and PLINK (34).

\section{Generation of Stably Transduced BJAB Cell Lines}

BJAB cell lines, each expressing one of the rs1131769 variants of interest, were created using custom suCMV promoter-based lentivectors containing a Blasticidin resistance gene (GenTarget Inc.; San Diego, CA). Lentiviral particles were produced in 293T packaging cells using the SureTiter ${ }^{\mathrm{TM}}$ Lentiviral vector system (GenTarget Inc.; San Diego, CA), in DMEM with 10\% FBS at approximately $10^{7}$ IFU/per $\mathrm{ml}$. Transduction with lentiviral particles was performed at MOI of 10 in the presence of Polybrene (Millipore Sigma) at $8 \mu \mathrm{g} / \mathrm{mL}$, and stable cell clones were selected for using Blasticidin (InvivoGen; San Diego, CA) at $10 \mu \mathrm{g} / \mathrm{mL}$.

\section{Transfection of Cells With STING Variants}

For HEK 293 T cells, transfection was performed with $20 \mathrm{ng}$ of the STING plasmid constructs (pUNO1-hSTING-H232 and pUNO1-hSTING-WT, InvivoGen; San Diego, CA) and with 20 ng cGAS (pUNO1-hcGAS, InvivoGen; San Diego, CA) using Lipofectamine LTX and PLUS ${ }^{\mathrm{TM}}$ Reagent (Invitrogen; Carlsbad, CA), according to the manufacturer's instructions.

\section{Cellular Stimulation With STING Ligands}

STING allele-expressing BJAB cells were plated at 300,000 cells/ $\mathrm{mL}, 0.5 \mathrm{~mL} /$ well in a 24 -well plate. $50 \mathrm{ug} / \mathrm{mL}$ of 2'3' cGAMP or $\mathrm{ddH} 2 \mathrm{O}$ were added to stimulated and mock-stimulated wells, respectively. Stimulated and control cells were harvested at the indicated times after 2'3' cGAMP stimulation and centrifuged $5 \mathrm{~min}$ at 5,000 rpm in microcentrifuge tubes. Supernatants were collected and frozen for ELISA analysis. Cells were resuspended in $200 \mathrm{uL}$ RNAProtect (Qiagen; Valencia, CA) and frozen at $-20^{\circ} \mathrm{C}$.

\section{Protein Phosphorylation (Western Blot)}

Protein expression and phosphorylation (for IRF3 and TBK1) was assessed in transiently transfected HEK $293 \mathrm{~T}$ cells and lentivirus-transduced stable $\mathrm{BJAB}$ cell lines, expressing the STING alleles of interest. For protein expression and western blotting experiments, cells were incubated overnight in antibiotic-free medium and then stimulated with 2'3'cGAMP (Invivogen; $20 \mu \mathrm{g} / \mathrm{mL}$ for the HEK $293 \mathrm{~T}$ cells and $100 \mu \mathrm{g} / \mathrm{mL}$ for the BJAB cells) for different timepoints ( $15 \mathrm{~min}, 30 \mathrm{~min}, 1 \mathrm{~h}, 2 \mathrm{~h}$, and $4 \mathrm{~h}$ ). The cells were lysed in RIPA lysis buffer (Sigma) containing protease and phosphatase inhibitors (protease inhibitors cOmplete ${ }^{\mathrm{TM}}$ and phosphatase inhibitors PhosSTOP $^{\mathrm{TM}}$, Roche). Lysates were centrifuged at 16,000 RPM and $4^{\circ} \mathrm{C}$ for $20 \mathrm{~min}$. Protein concentrations were quantified using the Pierce BCA Protein Assay Kit (ThermoFisher Scientific; Minneapolis, $\mathrm{MN}$ ), and equal protein amounts (2 to $3 \mu \mathrm{g}$ ) were used for western blot analysis. Laemmli buffer (Bio-Rad; Hercules, CA) with $\beta$-mercaptoethanol was added to the samples, and the lysates were denatured by incubating at $95^{\circ} \mathrm{C}$ for $5 \mathrm{~min}$ and were centrifuged at $16,000 \mathrm{RPM}$ for $1 \mathrm{~min}$. Samples were loaded onto 4-20\% Criterion ${ }^{\mathrm{TM}}$ gels (Bio-Rad; Hercules, CA), and then proteins were transferred to TransBlot $^{\circledR}$ Turbo Midi PVDF membranes (Bio-Rad; Hercules, CA) using the Trans-Blot ${ }^{\circledR}$ Turbo $^{\mathrm{TM}}$ Transfer System (Bio-Rad; Hercules, CA). Blots were blocked with 3\% BSA and probed overnight (at $4^{\circ} \mathrm{C}$ ) with primary monoclonal rabbit anti-STING (cat. \# 13647), anti-TANK-binding kinase 1 (TBK1, cat. \# 3504), anti-pTBK1 (cat. \# 5483), anti-interferon regulatory factor 3 (IRF3, cat. \# 4302), and anti-pIRF3 antibody (cat. \# 4947) (all from Cell Signaling Technologies; Beverly, MA), or mouse monoclonal anti-alpha tubulin antibody (cat. \# 40742, Abcam; Cambridge, MA) for loading control. Membranes were washed and incubated for $1 \mathrm{~h}$ at room temperature with the appropriate HRP-labeled pre-absorbed goat anti-rabbit (cat. \# sc-2054) or anti-mouse (cat. \# sc-2055) secondary antibodies (Santa Cruz Biotechnology, Inc.; Dallas, TX). The membranes were washed, developed using Clarity Western ECL Substrate Solution (BioRad; Hercules, CA) for $10 \mathrm{~min}$, and imaged using the ChemiDoc $^{\mathrm{TM}}$ Touch Gel Imaging System (Bio-Rad; Hercules, CA). Comparisons were assessed using Student's t-test.

\section{Gene Expression (qPCR)}

Total RNA was extracted from frozen cells using Qiagen RNeasy Plus mini kits according to the manufacturer's instructions, and RNA concentrations were normalized between samples. Random-primer reverse transcription was done using RT2 First Strand kits (Qiagen; Valencia, CA), including a genomic DNA removal treatment, according to the manufacturer's instructions. qPCR was then done on each sample using the Qiagen RT2 SYBR Green/ROX qPCR Mastermix system using the following primers (35): IFN- $\alpha$, 5'-AAATACAGCCCTTG TGCCTGG-3'and 5'-GGTGAGCTGGCATACGAATCA-3'; IFN- $\beta$, 5'-AAGGCCAAGGAGTACAGTC-3' and 5'-ATCTT CAGTTTCGGAGGTAA-3'; IFN- $\lambda 1,5$ '-CGCCTTGGAAG AGTCACTCA-3'; IFN- $\lambda 1$ 5'-GAAGCCTCAGGTCCCAATTC3'; b-actin, 5'-AAAGACCTGTACGCCAACAC-3'; b-actin 5'GTCATACTCCTGCTTGCTGAT-3'; STING, Commercial Qiagen RT2 qPCR Primer Assay for Human TMEM173, MxA, Commercial InvivoGen IFNr qRT-Primer set, hOAS1-F and hOAS1-R; OAS1, Commercial InvivoGen IFNr qRT-Primer set, hMX1-F and hMX1-R. Quantitative PCR was done using an ABI ViiA-7 machine at the standard qPCR conditions starting with incubation at $95^{\circ} \mathrm{C}$ for $10 \mathrm{~min}$, followed by 40 cycles of $95^{\circ} \mathrm{C}$ for $15 \mathrm{~s}$ and $60^{\circ} \mathrm{C}$ for $1 \mathrm{~min}$. Ct values were normalized to $\beta$-actin levels and unstimulated controls by the standard $2 \Delta \Delta \mathrm{CT}$ method. Experimental conditions were compared using Student's t-test.

\section{Promoter Reporter Assays}

The promoter reporter assays were performed in HEK $293 \mathrm{~T}$ cells, stably expressing one of the STING alleles of interest (for rs1131769 - WT/R232 and H232) under blasticidin selection (36). We used pNiFty2-IFNB-SEAP and pNiFty2-56K-SEAP 
promoter-reporter plasmids (InvivoGen; San Diego, CA), encoding the INF $\beta$ minimal promoter and the ISG-56K promoter, respectively. Co-expression with constitutively activated IRF3 (or IRF7) leads to promoter induction measured by the inducible expression of the secreted embryonic alkaline phosphatase (SEAP) reporter gene. Promoter assays were performed as previously described (36) but with some modifications. Briefly, $2.5 \times 10^{4}$ cells per well (stably expressing STING alleles of interest under blasticidin selection) were cultured overnight in 96-well plates in antibioticfree medium [DMEM (Gibco Invitrogen Corporation; Carlsbad, CA)], containing 10\% fetal bovine serum (FBS, HyClone; Logan, UT). On the following day, cells were transfected with Lipofectamine ${ }^{\circledR}$ LTX (Invitrogen; Carlsbad, CA), according to the manufacturer's protocol, using a constant amount of reporter plasmid (100 ng of either pNiFty2-IFNB-SEAP or pNiFty2-56KSEAP per well), $0.2 \mu \mathrm{L}$ PLUS $^{\mathrm{TM}}$ Reagent (Invitrogen; Carlsbad, CA) per well, and $0.25 \mu \mathrm{L}$ Lipofectamine LTX per well. After overnight incubation, the medium was switched to Opti-MEM (Gibco Invitrogen Corporation; Carlsbad, CA), and cells were stimulated with one of two STING ligands: 2'3' cGAMP $(100 \mu \mathrm{g} /$ $\mathrm{ml}$ ), or inactivated vaccinia virus (MOI of 10 ) at $37^{\circ} \mathrm{C}$ for different time periods. Promoter induction was measured by the SEAP reporter secretion (quantified at $620 \mathrm{~nm}$ following addition of Quanti-Blue ${ }^{\mathrm{TM}}$ media, Invivogen, per the manufacturer's instructions). Experimental conditions were compared using Student's t-test.

\section{ELISA Measurement of Secreted Type I and Type III IFNs}

IFN $\alpha$ and IFN $\lambda$ production by 2'3' cGAMP-treated STINGtransduced $\mathrm{BJAB}$ cells were measured in triplicate using commercial sandwich ELISA assay sets (IFNo: VeriKine-HS ${ }^{\mathrm{TM}}$ Human Interferon Alpha All Subtype ELISA Kit, PBL Assay Science; Piscataway, NJ, and IFN $\lambda$ : Human IL-29/IL-28B [IFNlambda 1/3] DuoSet ELISA set, R\&D Systems; Minneapolis, MN) according to the manufacturer's instructions. Standard protein samples were diluted in cell culture media for accurate standard curve construction and calculations. Recombinant IFNs were used as positive controls while cell culture media served as the negative control. Biological duplicate samples from each timepoint were each assayed in technical duplicate. The level of sensitivity for the IFN $\alpha$ and IFN $\lambda$ assays were 12.5 and $62.5 \mathrm{pg} / \mathrm{mL}$, respectively.

\section{Molecular Modeling}

The atomic structure of the cyclic dinucleotide binding domain of STING has been experimentally solved (37). As is common for crystallographic structures, mobile loops were not resolved in these structures. To initially place residues within these mobile loops, we used the SwissModel server (38) and template PDB structures 4QXP (39) (open conformation with inhibitor bound) and 4F5Y (40) (closed conformation with cdGMP bound). Mutations present in each template were reverted to WT amino acids according to the UniProt sequence of the canonical transcript (Q86WV6-1). Simulations were run for the apo (un-liganded), cdGMP, and cGAMP ligand states.
We used NAMD (41) and the CHARMM27 with the CMAP (42) force field for Generalized Born implicit solvent molecular dynamics (isMD) simulations using previously optimized conditions (43) that included the following: 1) an interaction cutoff of $15 \AA$; 2) strength tapering (switching) starting at $12 \AA ; 3$ ) a 1 fs simulation time step with conformations recorded every $2 \mathrm{ps}$; 4) an initial conformation that was energy minimized for 20,000 steps; and 5) heating to $300 \mathrm{~K}$ over 300 ps via a Langevin thermostat. From each of the 12 conditions (two initial conformations, two alleles, and three ligand states), 100ns of simulation trajectory was generated and the final 70ns analyzed. Three additional and independent 20ns replicates for each condition were generated using the same procedure. All trajectories were aligned to the initial R232 closed conformation using $\mathrm{C}^{\alpha}$ atoms. Trajectories were then evaluated using multiple metrics, including C-alpha Root Mean Squared Deviation (RMSD), Root Mean Squared Fluctuation (RMSF), Principal Component (PC) analysis, alignment-free distance difference matrix (44-46), and distance monitors across the ligand binding site. We quantified variance of atomic $C^{\alpha}-C^{\alpha}$ (Figure 7A) distances using median absolute difference (MAD). Analysis was performed using custom scripts on the Bio3D R package (46) and VMD (47).

\section{RESULTS}

\section{Overview of Cohorts and IFN $\alpha$ Response}

We conducted a GWAS meta-analysis on two cohorts $(n=1,076$ and 1,058$)$ of smallpox vaccine recipients $(11,12,15)$ with immune outcome data and genome-wide SNP-typing. We imputed additional SNPs, as described in Materials and Methods section. As the full dataset was available for all subjects, the cohorts were combined to increase statistical power, and a final study sample of 1,653 Caucasian subjects was available for analysis (see Supplementary Table 1 for demographic information). The actual cohort used in the analysis for each immune outcome varied depending on how many of those 1,653 individuals had data for that specific outcome. Our original intent was to determine whether or not there were genetic polymorphisms associated with markers of vaccine-induced cellular immunity, as had been noted in preliminary reports on the San Diego Cohort $(24,25,48)$. VACV was inactivated in order to minimize the immunomodulatory effect of poxvirus-encoded proteins and to allow full development of the cytokine response. Our outcomes of interest included both innate anti-viral outcomes (secretion of IL-1 $1 \beta$, IL-6, IFN $\alpha$ ) and markers associated with adaptive immune responses (neutralizing antibody titer, IFN $\gamma$ ELISPOT response in PBMCs, IFN $\gamma$ ELISPOT in CD8+ $\mathrm{T}$ cells, as well as secretion of IL-2, IFN $\gamma$, IL-12p40, and TNF $\alpha$ ).

Surprisingly, our GWAS analysis found only a strong signal on chromosome 5 associated with IFN $\alpha$ secretion that exceeded the genome-wide significance level (Figures 1A, B). There were two suggestive signals: one on chromosome 11 associated with the CD8+ $\mathrm{T}$ cell IFNg ELISPOT response and another on 
A

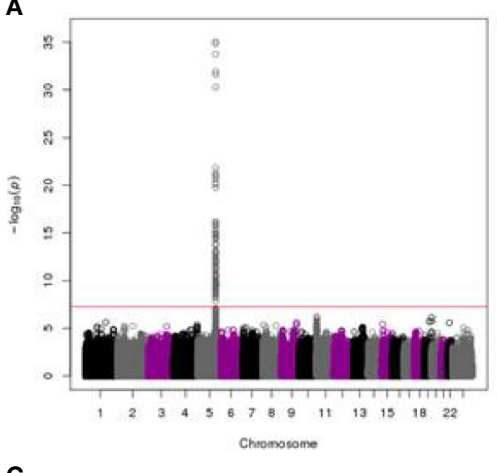

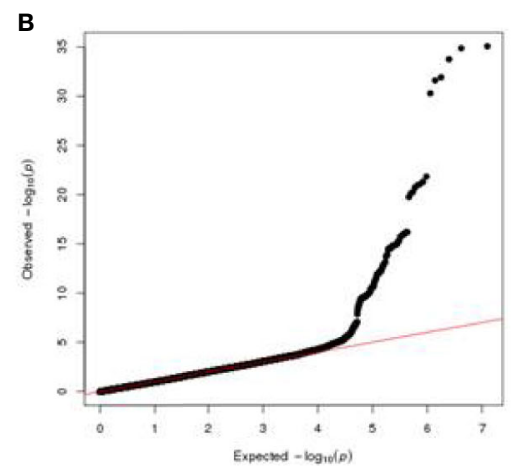

C

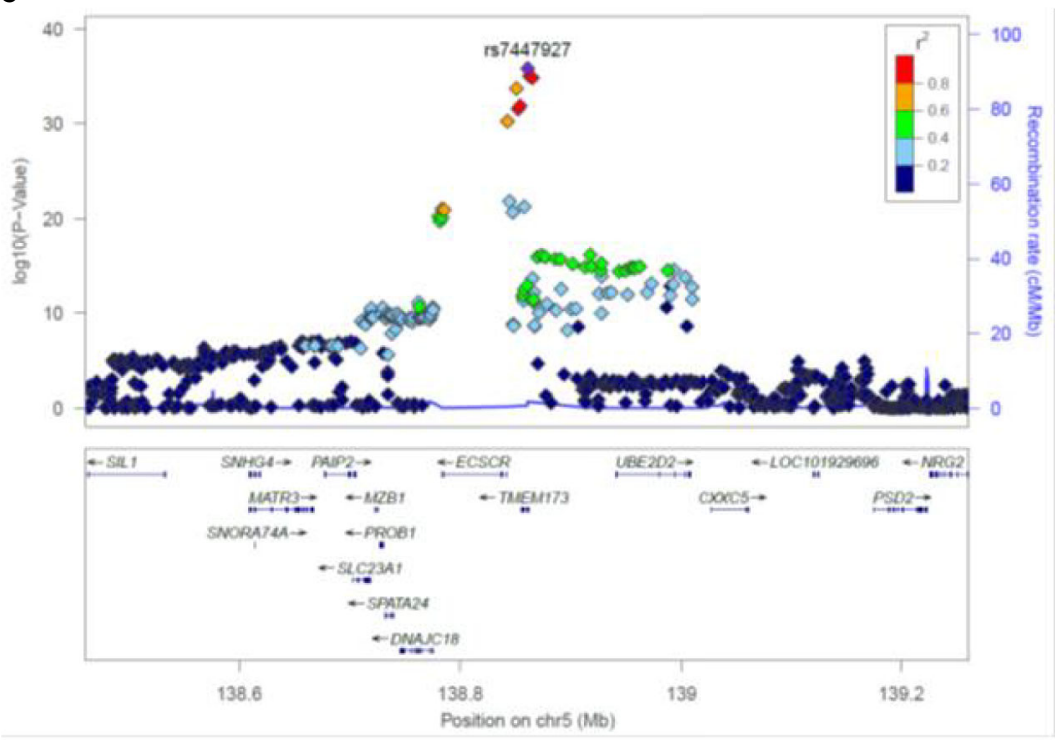

FIGURE 1 | GWAS genotyping results in the combined smallpox vaccine recipient cohort. (A) Manhattan plot indicating SNPS associated with IFN $\alpha$ response. (B) $\mathrm{QQ}$ plot of genome-wide p-values. (C) Locus-zoom plot depicting region on chromosome 5 with the strongest association signal. SNP LD is shown in color. The name and location of each gene is shown at the bottom of the panel.

chromosome 9 associated with IFN $\gamma$ secretion. The remaining outcomes were not associated with any genetic variants. Due to the strength of the IFN $\alpha$ signal and the fact that the other two potential signals did not reach a genome-wide threshold for significance, we focused our efforts on exploring the region on chromosome 5 associated with IFN $\alpha$ secretion.

The locus-zoom plot in Figure 1C depicts the SNPs with the strongest statistical association with IFN $\alpha$ response. We used a genome-wide threshold of $\mathrm{p}$-value $<5 \times 10^{-8}$ to establish statistical significance. Further details on the SNPs meeting this threshold are provided in Table 1. Of the 1,653 individuals with genotyping available, 1,605 also had IFN $\alpha$ secretion data. This cohort had a median IFN $\alpha$ secretion level of $126 \mathrm{pg} / \mathrm{mL}$ (IQR: 48.6-229.6) in PBMC cultures after vaccinia virus stimulation. As illustrated in Figure 2, TT homozygotes (H232 STING) had a median IFN $\alpha$ response of $17.7 \mathrm{pg} / \mathrm{mL}$, while individuals homozygous for the R232 STING allele (CC genotype) had an 8-fold higher response (143.6 pg/mL). Heterozygotes had an intermediate phenotype. We did not identify any SNPs in other genes (including those associated with the STING pathway, such as $c G A S$, TBK1, or $I R F 3)$ associated with variations in IFN $\alpha$ secretion.

Several of the SNPs significantly associated with IFN $\alpha$ secretion were located in TMEM173, which encodes for STING - an adaptor molecule mediating type I IFN responses to cyclic dinucleotides and double-stranded DNA. STING has previously been shown to play an important role in the innate immune response to poxviruses $(20,49)$.

A number of additional non-synonymous polymorphisms potentially affecting STING function have previously been identified, including R71H (rs11554776), G230A (rs78233829), R293Q (rs7380824), and R232H (rs1131769) (22, 50). In order to determine which SNPs in the haplotype contributed to the response, while accounting for correlation among SNPs, we used haplo.stats software in $\mathrm{R}$ to compute the haplotype frequencies for these four SNPs: rs11554776; rs78233829; rs1131769; and rs7380824 (51). 
TABLE 1 | Top SNPs significantly associated with vaccinia virus-specific IFN $\alpha$ secretion.

\begin{tabular}{|c|c|c|c|c|c|c|c|c|}
\hline SNP & ChromosomeLocation & Gene & SNPFunction & GeneLocation & p-value & Minorallele & Majorallele & MAF \\
\hline rs7447927 & 138861146 & TMEM173 & protein-coding & synonymous & $1.49 E-36$ & C & $\mathrm{G}$ & $34.7 \%$ \\
\hline rs13166214 & 138862744 & TMEM173 & protein-coding & 5'upstream & 8.92E-36 & A & $\mathrm{G}$ & $35.3 \%$ \\
\hline rs7444313 & 138865423 & TMEM173 & protein-coding & 5'upstream & $1.41 \mathrm{E}-35$ & $G$ & A & $34.5 \%$ \\
\hline rs13181561 & 138850905 & TMEM173 & protein-coding & 3'downstream & $1.81 \mathrm{E}-34$ & $G$ & $A$ & $30.0 \%$ \\
\hline rs55792153 & 138854203 & TMEM173 & protein-coding & 3'downstream & $1.26 \mathrm{E}-32$ & A & C & $34.2 \%$ \\
\hline rs13153461 & 138852369 & TMEM173 & protein-coding & 3'downstream & 2.61E-32 & $G$ & $A$ & $31.4 \%$ \\
\hline rs9716069 & 138842818 & ECSCR & protein-coding & 5'upstream & $5.32 E-31$ & $\mathrm{~T}$ & A & $31.3 \%$ \\
\hline rs28419191 & 138844599 & ECSCR & protein-coding & 5'upstream & $1.50 \mathrm{E}-22$ & $\mathrm{~T}$ & $\mathrm{C}$ & $13.2 \%$ \\
\hline rs1131769 & 138857919 & TMEM173 & protein-coding & missense & $5.25 E-22$ & $T$ & $c$ & $14.0 \%$ \\
\hline rs11954057 & 138783832 & RNU5B-4P & pseudo & 3'downstream & 8.99E-22 & C & $\mathrm{G}$ & $32.5 \%$ \\
\hline rs36137978 & 138785565 & ECSCR & protein-coding & 5'upstream & 1.13E-21 & $\mathrm{C}$ & A & $31.6 \%$ \\
\hline rs10875554 & 138847652 & ECSCR & protein-coding & 5'upstream & $1.99 E-21$ & A & C & $15.4 \%$ \\
\hline rs6596479 & 138780599 & RNU5B-4P & pseudo & 5'upstream & $5.63 E-21$ & $\mathrm{C}$ & $\mathrm{T}$ & $31.9 \%$ \\
\hline rs7446197 & 138783734 & RNU5B-4P & pseudo & 3'downstream & 7.51E-21 & A & $\mathrm{G}$ & $33.8 \%$ \\
\hline rs10463977 & 138781765 & RNU5B-4P & pseudo & 5'upstream & $1.80 E-20$ & $\mathrm{C}$ & $\mathrm{T}$ & $32.4 \%$ \\
\hline rs2434576 & 138917674 & UBE2D2 & protein-coding & 5'upstream & $6.57 \mathrm{E}-17$ & $G$ & A & $30.8 \%$ \\
\hline rs34530489 & 138873627 & LOC642262 & pseudo & gene & $7.00 \mathrm{E}-17$ & $G$ & $A$ & $31.4 \%$ \\
\hline rs35779874 & 138869847 & LOC642262 & pseudo & 5'upstream & $1.11 \mathrm{E}-16$ & A & $\mathrm{G}$ & $31.2 \%$ \\
\hline rs7378724 & 138876953 & LOC642262 & pseudo & gene & $1.13 \mathrm{E}-16$ & $G$ & $A$ & $30.9 \%$ \\
\hline rs78233829 & 138857925 & TMEM173 & protein-coding & missense & $3.16 \mathrm{E}-13$ & $\mathbf{G}$ & C & $17.6 \%$ \\
\hline rs11554776 & 138861078 & TMEM173 & protein-coding & missense & $1.05 \mathrm{E}-12$ & $\mathbf{T}$ & C & $16.5 \%$ \\
\hline rs7380824 & 138856982 & TMEM173 & protein-coding & missense & $1.25 E-12$ & $\mathbf{T}$ & C & $17.7 \%$ \\
\hline
\end{tabular}

MAF, minor allele frequency. Bold, italics - SNP studied in this report. Bold - SNPS in HAQ STING haplotype.

A

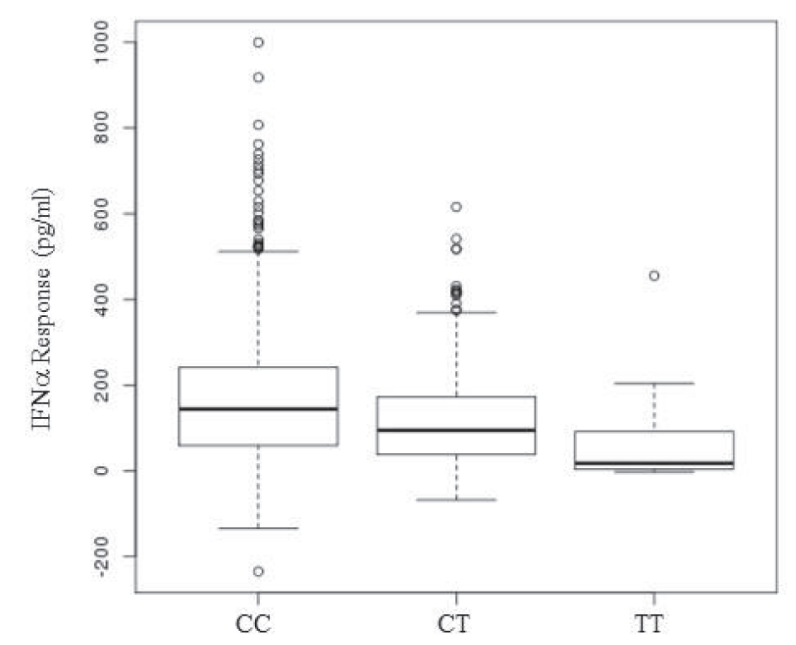

B

\begin{tabular}{|c|c|c|c|}
\hline & \multicolumn{3}{|c|}{ rs1131769 genotype } \\
\hline & $\mathrm{CC}$ & $\mathrm{CT}$ & $\underline{\mathrm{TT}}$ \\
\hline $\begin{array}{c}\text { Median IFN-alpha } \\
(\mathrm{pg} / \mathrm{mL})\end{array}$ & 143.6 & 93.6 & 17.7 \\
\hline
\end{tabular}

FIGURE 2 | IFNa response in smallpox vaccine recipients displays a dosedependent association with rs1131769. (A) Box and whisker plots for major allele homozygotes (CC), heterozygotes (CT), and homozygous minor allele (TT) subjects. The $\mathrm{C}$ allele corresponds to the R232 STING variant and the T allele corresponds to the H232 STING variant. (B) Median IFNa response (pg/ $\mathrm{mL}$ ) by rs1131769 genotype group.
The haplotype frequencies were very close between the U.S. and San Diego cohorts; therefore, we proceeded to use the combined sample to evaluate the association of haplotypes with IFN $\alpha$ response. The results presented in Table 2 illustrate the effects of the haplotypes on IFN $\alpha$ response and the frequencies of the haplotypes. We compare the haplotype CCTC (H232) with the most frequent haplotype CCCC (R232: treated as the baseline in the linear regression model) in order to focus on the effect of the T allele for rs1131769 while controlling for the effects of the other three SNPs on the haplotype, and identified a statistically significant $\left(\mathrm{p}<2 \mathrm{E}^{-16}\right)$ decrease in IFN $\alpha$ response. The contrast of the haplotype CCCT with the baseline was not statistically significant, but we had limited power for this comparison because of the low frequency of the haplotype CCCT. We also used likelihood ratio statistics to contrast the effects of the haplotypes CGCT and TGCT, which differed only at the first SNP position, but the effects of these haplotypes were not significantly different ( $\mathrm{p}$-value $=0.46$ ), but once again power was limited because of the rarity of haplotype CGCT. These analyses suggest that the SNP rs1131769 is likely the main variant in the haplotype impacting the association with IFN $\alpha$ response. However, the strong association of the haplotype TGCT with IFN $\alpha$ response suggests that there might be additional SNPs in the region that are in linkage disequilibrium with our measured SNPs that are also associated with IFN $\alpha$ response. To further explore this, we computed the dose of the minor allele for each of the four SNPs and performed backward regression, thereby ignoring haplotypes. The two SNPs rs7380824 and rs1131769 remained in the model (each with $\mathrm{p}$-value $<2 \mathrm{E}^{-16}$ ), illustrating that each SNP is strongly associated with IFN $\alpha$ response after adjusting for the other SNPs. The other two SNPs, rs78233829 
TABLE 2 | The Effect of rs1131769 on IFN $\alpha$ Response is Independent of the Effect Mediated by the HAQ Haplotype.

\begin{tabular}{|c|c|c|c|c|}
\hline Term in Model & Regression Coefficient ${ }^{\star \star}$ & Standard Error of Coefficient & p-value ${ }^{\star \star \star}$ & Haplotype Frequency \\
\hline Intercept & 0.31 & 0.037 & $<2 \mathrm{E}-16$ & \\
\hline Cohort US vs. SD & 0.037 & 0.044 & 0.40 & \\
\hline \multicolumn{5}{|l|}{ Haplotypes* } \\
\hline $\operatorname{cCCC}$ & Baseline & & & 0.683 \\
\hline CCCT (H232) & -0.011 & 0.307 & 0.97 & 0.002 \\
\hline CCTC (Q293) & -0.546 & 0.045 & $<2 \mathrm{e}-16$ & 0.139 \\
\hline CGCT (AQ) & -0.281 & 0.146 & 0.05 & 0.011 \\
\hline TGCT (HAQ) & -0.409 & 0.042 & $<2 \mathrm{e}-16$ & 0.164 \\
\hline
\end{tabular}

*TMEM173 SNPs (haplotype) from left to right: rs 11554776 (encodes amino acid/AA change at position 71 ) —rs78233829 (encodes AA change at position 230 ) - rs 1131769 (encodes AA change at position 232) - rs 7380824 (encodes AA change at position 293). In bold are designated the minor alleles defining the respective haplotypes, and in italics are designated the commonly used names of the haplotypes (that are based on encoded amino acid/acids and their position).

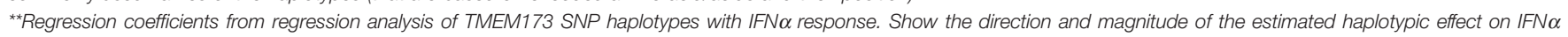
response compared to the haplotype (CCCC) with the greatest population frequency.

${ }^{* * *} P$-value from regression analysis.

and rs11554776, were not statistically significant and were both strongly correlated with rs7380824 (Pearson correlations > 0.95).

\section{Promoter Activity of rs1131769 Variants}

Two plasmids expressing the secreted embryonic alkaline phosphatase (SEAP) reporter gene, under control of either the INF $\beta$ promoter or the interferon stimulated gene (ISG)-56K promoter, were used to measure ligand-stimulated promoter activity in HEK293T cells expressing either R232 or H232 STING (Figure 3). Both 293T variant cell lines expressed high levels of STING mRNA (Figure 3D) with no significant difference between alleles. Upon 2'3' cGAMP stimulation, IFN $\beta$ promoter induction was significantly higher at $10 \mathrm{~h}$ poststimulation in R232 cells compared to cells expressing H232 (Figure 3A, p=0.02). Similarly, we observed statistically significant higher induction of the ISG-56K promoter activity in R232 upon stimulation with either 2'3' cGAMP at $4 \mathrm{~h}$ and $8 \mathrm{~h}$ post-stimulation (Figure 3B, $\mathrm{p}=0.006$ and $\mathrm{p}=0.004$, respectively) or inactivated vaccinia virus at $8 \mathrm{~h}$ post-stimulation (Figure 3C, $\mathrm{p}=0.002$ ).

In vitro stimulation of our $\mathrm{H} 232$ and $\mathrm{R} 232$ STING-transfected cells lines with live vaccinia virus resulted in global
A

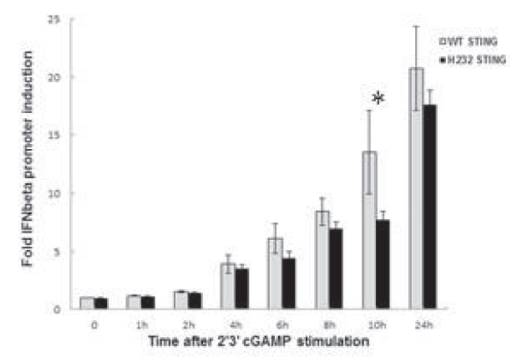

C

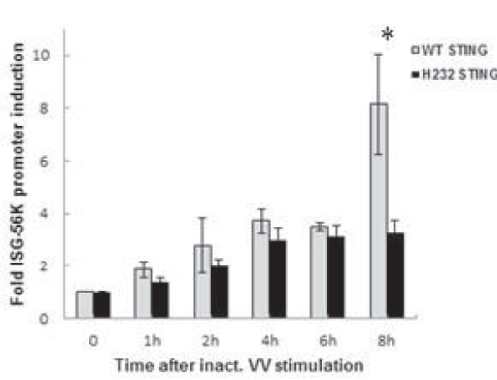

B

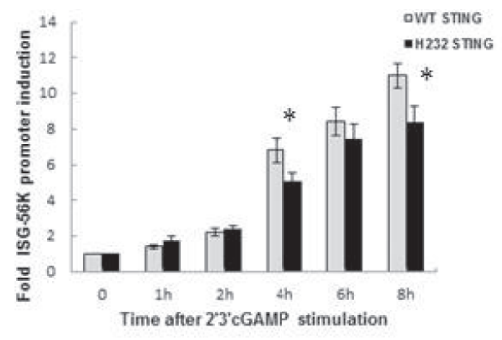

D

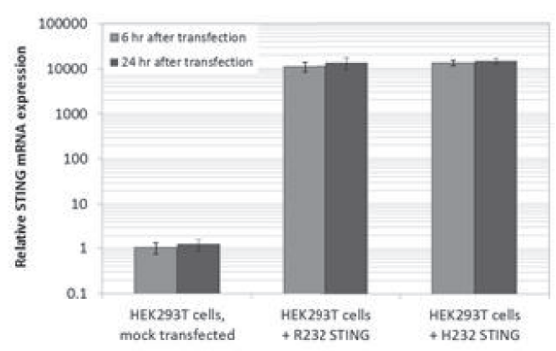

FIGURE 3 | IFN $\beta$ and ISG-56K Promoter Activity is Greater in HEK 293T Cells Stably Expressing the R232 STING Variant. (A) IFN $\beta$ promoter induction following cGAMP stimulation. (B) ISG-56K promoter activity after cGAMP stimulation. (C) ISG-56K promoter activity after stimulation with inactivated vaccinia virus (inact. W). Data points for (A), (B, C) show the means with error bars representing the standard deviations of three replicates. (D) STING expression in HEK293T stably expressing R232 or H232 STING. Data points for (D) show the means with error bars representing the standard deviations of eight replicates. A two-tailed t-test* detected significant differences; see text for $p$-values. Each experiment was performed twice with nearly identical results. 


\section{A}

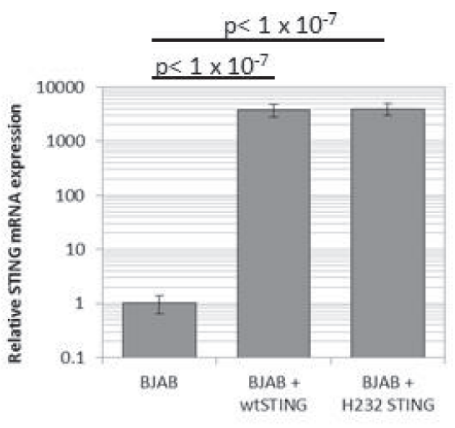

B

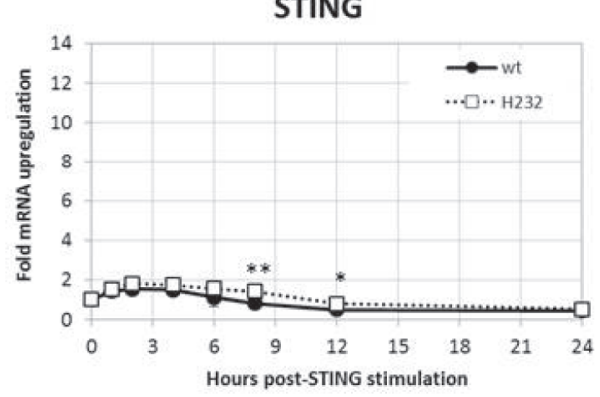

C

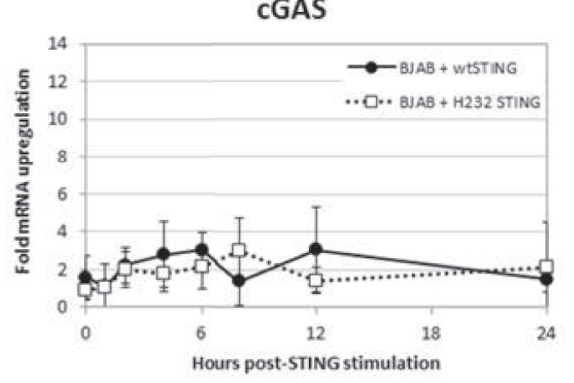

FIGURE 4 | Stable transfection of H232 or R232 STING into BJAB cells results in high level expression that is stable upon cGAMP stimulation. (A) Untransduced and stably transduced BJAB cell lines expressing STING alleles were harvested and assayed for STING mRNA using quantitative PCR. Values are shown as foldlevels relative to normal STING expression in untransduced BJAB cells. Data represent means and standard deviations of four biological replicates, assayed with technical duplicates. (B) Time course of TMEM173 expression in 2'3' cGAMP-stimulated (50ug/ml) in transduced BJAB cells stably expressing STING variants. (C) Time course of cGAS expression in stable BJAB transfectants stimulated with cGAMP. Values are presented as fold-increases over mock-treated cells, normalized to $\beta$-actin loading controls. Data points are the average of 8 replicates coming from four biological duplicates. Each experiment was performed twice. Two-tailed t-test: ${ }^{*} p<0.05 .{ }^{*} p<0.005$.

downregulation of gene expression, preventing us from examining differential effects between the two STING variants. In order to avoid such confounding issues with viral infection, we stimulated cells with 2'3' cGAMP or inactivated vaccinia virus for all further experiments.

\section{Gene Expression of rs1131769 Variants}

mRNA was extracted from PBMCs of individuals homozygous for the CC genotype (R232) and the TT genotype (H232), and the two TMEM173 variants were PCR amplified and cloned into lentivirus expression vectors. BJAB cells were transduced with these vectors, creating stable cell lines that constitutively overexpress each STING variant. The PCR products and completed expression vectors were both sequenced to verify the insertion of the correct genetic variants. As illustrated in Figure 4A, stable transfectants express $>1,000$-fold higher (and comparable between the two variants) STING mRNA than normal BJAB cells. Expression of both TMEM173 variants transiently and minimally (less than 2-fold) increased after 2'3' cGAMP stimulation, indicating the STING protein levels of either variant are unlikely to be significantly affected by cGAMP treatment (Figure 4B). Finally, we found that MB21D1 (encoding cGAS, an essential upstream nucleotidyltransferase in the STING pathway that generates cyclic cGAMP) gene expression was not significantly different between the two variants (Figure 4C), indicating that signaling pathway function upstream of STING was not affected by the STING gene variants. Note that the HEK293T lines used in this report were also transfected with $M B 21 D 1$ as this cell line is known to be deficient in cGAS expression (52).

\section{Effect of rs1131769 Variants on Downstream Phosphorylation}

Transiently transfected HEK293T and stably transfected BJAB cells were stimulated with 2'3' cGAMP for the indicated time periods, and phosphorylation of downstream intermediates TBK1 and IRF3 was evaluated (Figures 5A, B). In transiently transfected HEK293T cells (also expressing cGAS), H232 STING expression was accompanied by a delay in phosphorylation of both TBK1 and IRF3 until 1 hour after stimulation. Interestingly, we observed substantial pTBK1 and pIRF3 baseline phosphorylation (at timepoint 0) for the R232 STING variant (but not for the $\mathrm{H} 232$ variant associated with diminished phosphorylation/activation), which is likely due to STING overexpression. The H232 STING expression at timepoints 0 and 30 min was slightly reduced, but TBK1 and IRF3 protein 


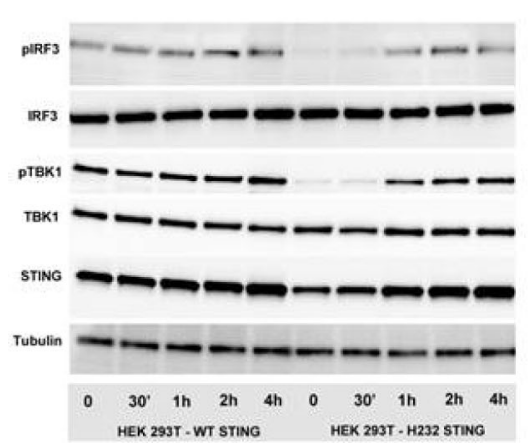

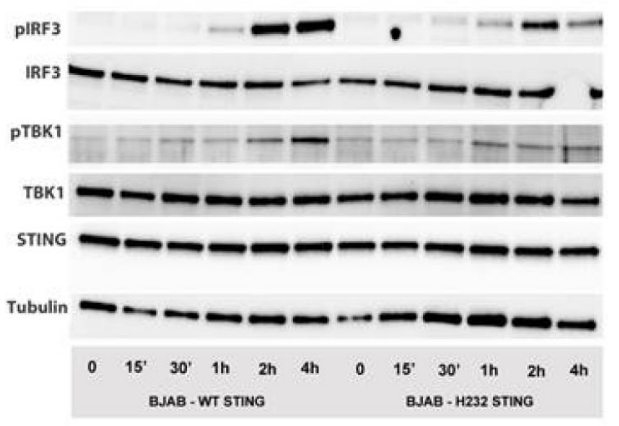

FIGURE 5 | Phosphorylation of IRF3 and TBK1 is delayed and decreased in the presence of H232 STING. (A) STING pathway activation (phosphorylation of IRF3 and TBK1) after 2'3' cGAMP stimulation of HEK 293 T cells, transiently expressing WT or H232 STING variants/alleles and cGAS. (B) STING pathway activation (phosphorylation of IRF3 and TBK1) after 2'3' cGAMP stimulation of lentivirus-created BJAB cell lines, stably expressing WT or H232 STING variants under Blasticidin selection. Each experiment was conducted three times with two biological duplicates per sample.

expression were similar between the two variants, which demonstrates that the observed differences in phosphorylation/ activation are valid. In the stably transduced BJAB line, the delayed phosphorylation was observed with both STING variants and the pattern/kinetics of phosphorylation was similar; however, the magnitude of TBK1 and IRF3 phosphorylation was significantly reduced (in particular at $2 \mathrm{~h}$ and $4 \mathrm{~h}$ poststimulation) in cells expressing $\mathrm{H} 232$ compared to cells expressing R232 STING. No major differences in STING protein levels, or in the unphosphorylated forms of either TBK or IRF3, were observed between the cells expressing the two STING variants at the observed timepoints.

\section{Effect of rs1131769 Variants on IFN Response}

Reasoning that differences in TBK1 and IRF3 phosphorylation between these variants should have downstream consequences, we decided to examine differential pathway activity mediated by the two STING variants. We stimulated R232 and H232 STING variant-expressing stable BJAB cell lines with 2'3' cGAMP and measured gene expression ( $\mathrm{qPCR}$, Figure 6A) and protein secretion (ELISA, Figure 6B) over time. cGAMP stimulation induced both type I (IFN $\alpha$, IFN $\beta$ ) and type III (IFN $\lambda 1)$ interferons, with significantly higher levels of IFNs (mRNA and protein) observed in R232 cells. This effect was consistent regardless of the cGAMP isomer used for stimulation (Supplementary Figure S1). Furthermore, the expression of the classical antiviral ISGs, MX1 and OAS1, after 2'3'cGAMP stimulation confirmed the greater STING pathway activation in R232 STING-expressing cells over the H232 STING-expressing cells (Figure 6C).

\section{Molecular Modeling of STING Variants}

In order to begin elucidating the mechanism underlying the greater STING activity in R232-expressing cells, we used molecular modeling and molecular dynamics simulations to examine structural and functional differences between the R232 and H232 variants. H232 exhibited larger overall deviation from the initial experimental structure, as quantified by RMSD (Figure 7A). This greater deviation (a reflection of mobility) occurred both in the presence or absence of ligand. As RMSD is a global measure, we also quantified per-residue mobility using RMSF (Figure 7B), which indicated that the ligand-binding loops were more mobile for H232 compared to R232, particularly when the 2'3' cGAMP ligand was bound. We further quantified the displacement of the ligand-binding loops using simple distance measures between residue 232 in each monomer. Regardless of the presence or absence of cGAMP, the ligand-binding loops of H232 were further separated from each other (Figure 7C) and from the base of the ligand-binding site (Figure 8) compared to R232. Our initial simulations assumed that the ligand-binding loops were closed over the base of the ligand-binding site; structures displayed in Figures 7D, E highlight the difference in STING conformation between H232 and R232. Simulations assuming an open ligand-binding loop conformation observed the same effect of H232 compared to R232 (Supplementary Figure S2). In summary, H232 exhibited greater structural flexibility and mobility of the ligand-binding loops in both the open or closed conformations and in the presence or absence of cGAMP.

\section{DISCUSSION}

Our GWAS across two cohorts of smallpox vaccine recipients, totaling just over 1,600 individuals, identified a highly significant $\left(\mathrm{p}<1 \times 10^{-30}\right)$ association signal from a region on chromosome 5 that was linked to significant inter-individual variations in IFN $\alpha$ response to in vitro stimulation with vaccinia virus. The lack of genetic association with vaccine response markers (e.g., neutralizing antibody titer and IFNg ELISPOT) indicates that the signal observed is likely reflective of an innate response to 
A
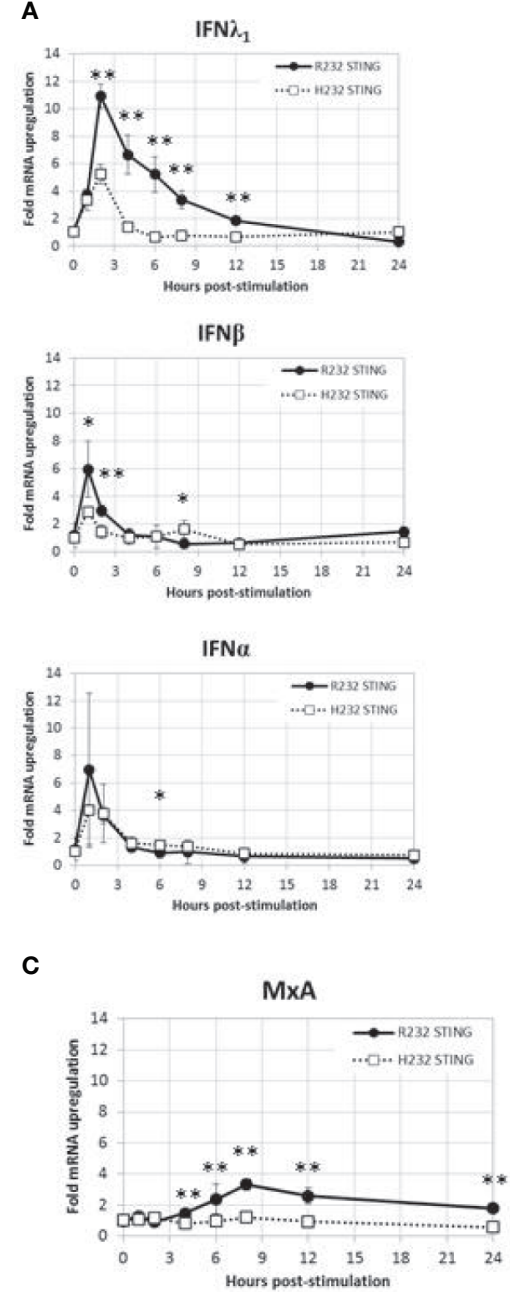

B

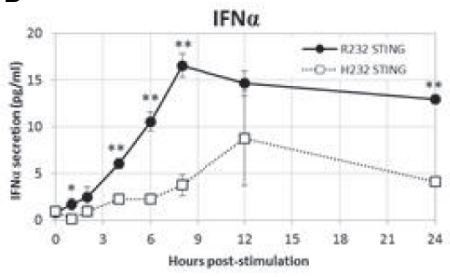

IFN $\lambda 1$
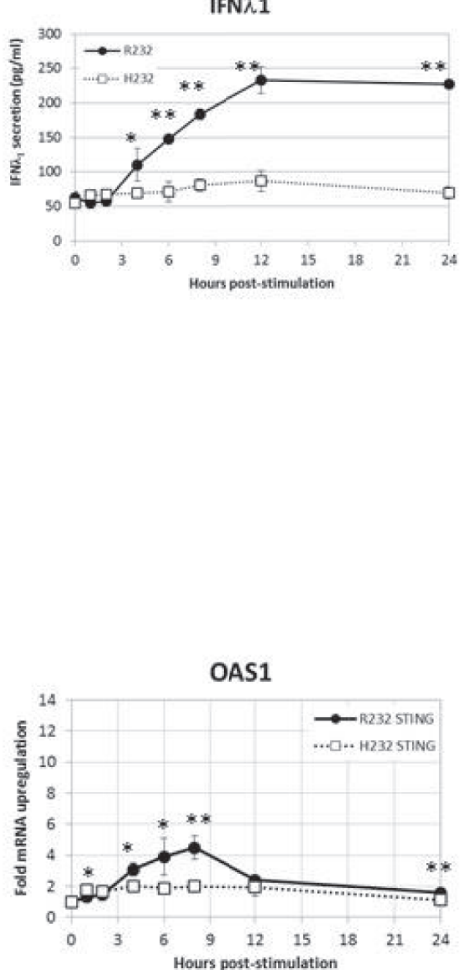

FIGURE 6 | WT and H232 STING-mediated IFN response in stably transduced BJAB cells. (A) Time course of IFNA, IFNB, IFNL1 gene expression after cGAMP stimulation. (B) Time course of cytokine secretion after CGAMP stimulation. (C) Activation of representative interferon-stimulated genes after cGAMP stimulation. All data points in (A, C) are means and error bars representing the standard deviations of total of eight replicates coming from four biological duplicates. For cytokine secretion (B) biological duplicate samples from each timepoint were each assayed in duplicate for a total of four replicates. Each experiment was performed twice. Two-tailed t-test: ${ }^{*} p<0.05 .{ }^{*} p<0.005$.

poxvirus infection rather than a $\mathrm{T}$ cell response. This suggests that our findings are broadly applicable to the response to poxvirus infection rather than smallpox vaccination.

Fine-mapping analysis identified a number of putative causal SNPs, including several in TMEM173, which encodes for the signaling adaptor protein STING. STING mediates IFN responses to dsDNA and cyclic dinucleotides through a pathway involving cGAS and the phosphorylation of TBK1 and IRF3. Our regression modeling also indicated that multiple SNPs within TMEM173 have independent effects on the phenotypic outcome. Homozygotes for the H232 allele of rs1131769 in TMEM173 exhibit a 90\% reduction in IFN $\alpha$ secretion compared to R232 homozygotes. This is a highly significant effect that may have significant downstream consequences for poxvirus immunity. We have previously reported that a small percentage of smallpox vaccine recipients have impaired innate immune responses to vaccinia virus and that these same individuals also have suboptimal cellular immunity (13). Our current results provide additional support to existing data $(53,54)$ suggesting that appropriate innate immune responses are necessary for robust adaptive immunity to vaccinia virus. Further investigation of this effect on smallpox immunity is warranted.

We conducted a series of experiments with the intention of elucidating functional effects of this SNP that might be underlying the identified genotype-phenotype association. We assessed gene expression of both variants of TMEM137 by PCR and STING expression by western blot and did not detect significant differences between variants in either gene or protein expression. We observed higher promoter activity of 

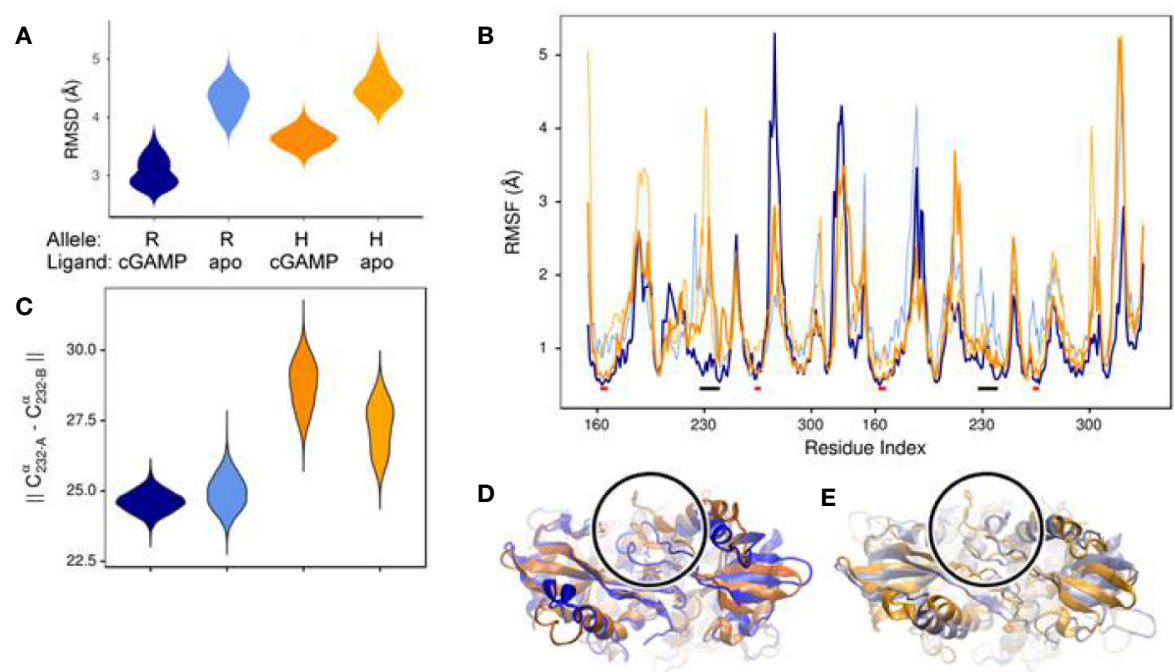

FIGURE 7 | Molecular Simulation ligand binding loop contact with cGAMP in R232 (wt) and H232 STING. (A) H232 consistently showed greater deviations from the initial open conformation, both in the presence and absence (marked as: "apo") of cGAMP. Simulation data from R232 is shown in blue, H232 in orange, and cGAMP-bound forms are in a darker shade. This color coding is continued throughout each panel. (B) Residues within the ligand binding loops, indicated by black bars, show less difference in mobility between unbound and cGAMP-bound forms. The ligand binding site residues, indicated by red bars, are more comparable in their mobility. (C) We monitored the distance between residue 232 from each monomer of the STING dimer as a measure of the separation of the ligand binding loops starting from the closed loop conformation. The separation was greater for H232, compared to R232, in both the unbound and cGAMP-bound forms. (D) Representative conformations from the end of cGAMP-bound simulations are shown and the ligand binding loop circled and residue 232 (shown in ball-and-stick representation). The altered conformation of H232 is evident. (E) Similar changes to the ligand binding loop conformation were observed in the absence of cGAMP.

downstream IFN-inducible genes in the R232 variant compared to the $\mathrm{H} 232$ variant, which suggests that there are differences in activation of the STING pathway. Upon stimulation with 2'3' cGAMP, the R232 STING variant elicited faster phosphorylation of both TBK1 and IRF3 as well as resulted in a greater quantity of phosphorylated TBK1 and IRF3 in the cells. These changes led to a significant increase in IFN and IFN-stimulated gene expression in R232-expressing cells, confirming that the statistical association was rooted in differential biological activity. As is true of most transfection systems, the TMEM173 gene was overexpressed in our cell lines, with the HEK293T cells expressing 10,000 times as much TMEM173 as untransfected cells. The $\mathrm{BJAB}$ transfectants also expressed high levels of TMEM173, but the overexpression was an order of magnitude lower. More relevant to our results, protein expression was similar to the expression levels of endogenous IRF3, TBK1, and tubulin, suggesting that protein expression was within normal limits despite the upstream overexpression of STING observed at the gene level. We note that the expression (at the gene and protein level) of both variants was consistent; therefore, differences in activity are not a result of differential gene or protein expression between the variants.

Multiple genetic variants of TMEM173 have been described, including three non-synonymous SNPs: rs1131769 (H232R), which was the focus of our study; rs11554776 (R71H); and rs7380824 (R293Q). R71H and R293Q, together with a fourth SNP, rs78233829 (G230A), form the HAQ haplotype (22). Zhang et al. have previously reported that expression of the H232 variant results in reduced IFN $\beta$ transcription (55). Our results confirm and extend these initial findings, demonstrating that IFN $\alpha$ secretion is also affected, as is the expression of multiple interferon-stimulated genes. Regarding the 3 alleles in the HAQ haplotype, there has been some controversy over the biological effects of these alleles $(56,57)$. In one study, cells carrying the G230 variant had fully functional STING activity and the HAQ haplotype effect was attributed to the R71H and R293Q SNPs (50). A similar study evaluating TMEM173 variants found that the $\mathrm{R} 232 \mathrm{H}, \mathrm{R} 293 \mathrm{Q}$, and $A Q(\mathrm{G} 230 \mathrm{~A}, \mathrm{R} 293 \mathrm{Q})$ variants had minimal effects on endogenous STING activity while the reduced STING function of the HAQ (R71H, G230A, R293Q) haplotype was attributed to the $\mathrm{R} 71 \mathrm{H}$ variant (22). Our analysis supported previous findings that possession of the HAQ haplotype leads to reduced STING activity, demonstrated that the H232 variant also leads to reduced STING activity, and verified that this functional effect is independent of the $H A Q$ haplotype. Thus, our haplotype and regression model results indicate that multiple SNPs/haplotypes are independently associated with variations in IFN $\alpha$ secretion. Our study also provides a potential biochemical mechanism for the reduced IFN $\alpha$ activity mediated by the R232H variant; however, further work will be required to tease apart the contributions of each individual SNP to the resulting immune response phenotype.

The crystal structure of STING has been resolved, as has the structure of the H232 variant bound to cGAMP (58). Our molecular modeling simulations, using these structure data, revealed that the ligand-binding loops of STING were more 
A

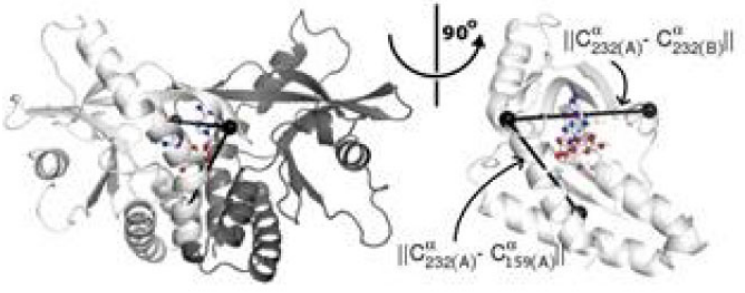

B
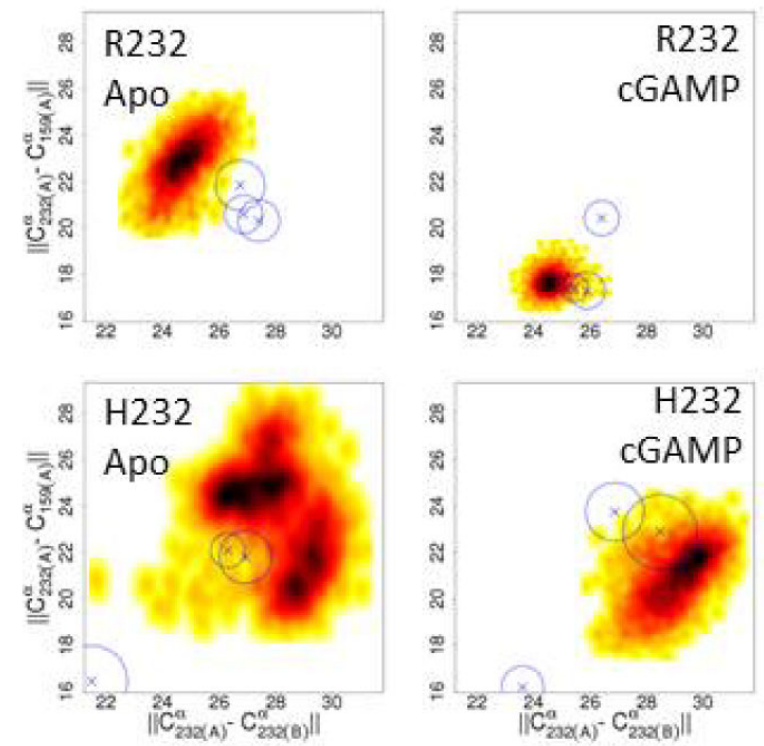

FIGURE 8 | Changes in the ligand binding loop between R232 and H232 are consistent across conditions. (A) Diagram of the STING structure showing one monomer of the closed conformation in white and the second monomer in gray, with the three $\mathrm{C}^{\alpha}$ atoms used to compute distances marked by black spheres. The bound cGAMP is shown as a ball-and-stick representation. A rotated view, with the second monomer omitted for clarity, more clearly shows the relationship between these $C^{\alpha}$ positions and the ligand binding site. (B) H232 lead to a consistent expansion of the distances between the ligand binding loops and from the ligand binding loops to the base of the ligand binding site. Probability density plots are shown with the most frequently sampled regions colored black and scaling through red and yellow as data become more sparse. The medians for additional independent replicates are indicated by Xs and a circle indicating the level of variability.

mobile for H232 compared to WT; that is, H232 loop conformations were more open and flexible than R232, even in the presence of bound cGAMP. We speculate that this may reflect a failure of the ligand-binding loops to either stay closed when beginning from a closed conformation, or to close when beginning from an open conformation. This may be indicative of weaker binding (and/or faster disassociation) between cGAMP and the H232 variant of STING. Our data suggest that the alterations in loop dynamics and weaker affinity of H232 STING for its ligand are the underlying molecular mechanism for the reduced STING activity that we observed for this variant. This hypothesis will require additional experimental data to confirm or refute.
With regard to poxviruses, it has been demonstrated that Modified Vaccinia Ankara (MVA) infection of dendritic cells (DCs) triggers type I IFN production through a STING and cGAS-dependent pathway involving cyclic dinucleotides (49). This type I IFN response is not seen during wild type vaccinia virus infection, likely due to the presence of viral immunomodulatory proteins such as C6L, E3L, and N1L in the wild type virus but not in MVA $(20,21)$. A recent report confirmed that MVA activated IRF3 in a cGAS- and STINGdependent manner, whereas wild type vaccinia strains failed to do so (19). Our experiments found clear differences in R232 and H232 STING activity in the presence of cyclic dinucleotides and inactivated vaccinia virus. It is possible that possession of the H232 STING variant may alter the effects of viral immunomodulation of this innate immune pathway during infection. This may happen through differential interactions with viral proteins, or indirectly as reduced secretion of type I IFNs may render viral immunomodulation more effective.

We have demonstrated that carriage of the $H$ variant of rs 1131769 results in a 90\% decrease in innate immune response (secreted IFN $\alpha$ ) to vaccinia virus. This data helps resolve prior conflicting reports regarding functional effects of STING polymorphisms. We hypothesize that the effect of this polymorphism is due to different flexibility/mobility in STING H232 loop conformations, which results in reduced ability of H232 STING to phosphorylate downstream signaling intermediates and mediate effective STING pathway activation.

Poxviruses represent a continuing public health concern due to the risk of bioterrorism use, zoonotic outbreaks (e.g., monkeypox, buffalopox, vaccinia-like viruses, and novel poxviruses), the increasing use of poxviruses for oncolytic viral therapy, and their use as vectors for vaccine antigens against HIV, rabies, Ebola, Zika, and other pathogens. STING also plays an essential role in triggering protective innate responses to DNA viruses (e.g., poxviruses, herpes simplex viruses, varicella zoster, EBV, HPV, and others) and multiple bacterial pathogens. Polymorphisms that reduce the effectiveness of the innate response to these threats are likely to enhance disease susceptibility and may have a deleterious effect on vaccine immunogenicity in the $\sim 15 \%$ of the population with this genotype. Given the broad potential impact of this pathway, this is an area that merits additional investigation.

Understanding how genetic factors control the immune response to poxviruses will have important clinical implications in how, when, and in whom these vectors can be safely and effectively used. Furthermore, this information may inform the use of adjuvants to overcome this defect and enhance vaccine responses or the development of therapeutic drugs that can be used to enhance the innate antiviral response during an infection.

\section{DATA AVAILABILITY STATEMENT}

The datasets presented in this study can be found in online repositories. The names of the repository/repositories and 
accession number(s) can be found below: https://immport.niaid. nih.gov, SDY28.

\section{ETHICS STATEMENT}

The studies involving human participants were reviewed and approved by Institutional Review Boards of the Mayo Clinic (Rochester, MN) and the Naval Health Research Center (NHRC; San Diego, CA). The patients/participants provided their written informed consent to participate in this study.

\section{AUTHOR CONTRIBUTIONS}

RK contributed to the conception and design of the study; participated in the acquisition of data and the analysis and interpretation of the results; prepared the initial draft; revised draft for intellectual content. IH contributed to the study design; participated in data acquisition and interpretation of study results; assisted in drafting the manuscript; revised draft for intellectual content; approved final version. IO contributed to the study design; participated in data acquisition and interpretation of study results; assisted in drafting the manuscript; revised draft for intellectual content; approved final version. EV contributed to the study design; participated in data acquisition and interpretation of study results; assisted in drafting the manuscript; revised draft for intellectual content; approved final version. BL contributed data analysis and interpretation; assisted in drafting the manuscript; revised draft for intellectual content; approved final version. DS supervised and contributed to the data analysis and interpretation; participated in drafting and revising the manuscript; approved final version. MZ contributed to the interpretation of study results, molecular modeling, data analysis, drafting the manuscript; provided critical review; approved final version. AO contributed to the design of the study; participated in data analysis and

\section{REFERENCES}

1. Fenner F, Henderson DA, Arita I, Jezek Z, Ladnyi ID. Smallpox and its Eradication. Switzerland: World Health Organization (1988) p. 1-1460 p.

2. Crotty S, Felgner P, Davies H, Glidewell J, Villarreal L, Ahmed R. Cutting edge: Long-term B cell memory in humans after smallpox vaccination. J Immunol (2003) 171(10):4969-73. doi: 10.4049/jimmunol.171.10.4969

3. Combadiere B, Boissonnas A, Carcelain G, Lefranc E, Samri A, Bricaire F, et al. Distinct time effects of vaccination on long-term proliferative and IFNgamma-producing T cell memory to smallpox in humans. J Exper Med (2004) 199(11):1585-93. doi: 10.1084/jem.20032083

4. Di Giulio DB, Eckburg PB. Human monkeypox: an emerging zoonosis. Lancet Infect Dis (2004) 4(1):15-25. doi: 10.1016/S1473-3099(03)00856-9

5. Essbauer S, Pfeffer M, Meyer H. Zoonotic poxviruses. Vet Microbiol (2010) 140(3-4):229-36. doi: 10.1016/j.vetmic.2009.08.026

6. Singh RK, Balamurugan V, Bhanuprakash V, Venkatesan G, Hosamani M. Emergence and reemergence of vaccinia-like viruses: global scenario and perspectives. Indian J Virol (2012) 23(1):1-11. doi: 10.1007/s13337-012-0068-1

7. Nagasse-Sugahara TK, Kisielius JJ, Ueda-Ito M, Curti SP, Figueiredo CA, Cruz AS, et al. Human vaccinia-like virus outbreaks in Sao Paulo and Goias interpretation; assisted in drafting the manuscript; revised the manuscript for intellectual content; approved the final version. GP contributed to the conception and design of the study; participated in data analysis and interpretation; secured funding for the project; participated in drafting and revising the manuscript; approved final version. All authors contributed to the article and approved the submitted version.

\section{FUNDING}

This study was supported by NIH through the NIAID Population Genetics Analysis Program Contract No.HHSN266200400065C and Contract No. HHSN272201000025C, and by the National Center for Research Resources grant 1 UL1 RR024150-01. The content is solely the responsibility of the authors and does not necessarily represent the official views of the National Institutes of Health.

\section{ACKNOWLEDGMENTS}

We gratefully thank the study subjects for their participation. We also thank Drs. Megan Ryan and Kevin Russell, as well as the clinical staff at the Naval Health Research Center for assistance with subject recruitment. We thank Nathaniel D. Warner and Krista M. Goergen for statistical and programming assistance and Caroline L. Vitse for proofreading and editorial assistance. This paper has been uploaded to the preprint server bioRxiv (doi: https://doi.org/10.1101/2020.05.28.1216570).

\section{SUPPLEMENTARY MATERIAL}

The Supplementary Material for this article can be found online at: https://www.frontiersin.org/articles/10.3389/fimmu.2020. 567348/full\#supplementary-material
States, Brazil: virus detection, isolation and identification. Rev Inst Med Trop Sao Paulo (2004) 46(6):315-22. doi: 10.1590/S0036-46652004000600004

8. Breman JG, Arita I, Fenner F. Preventing the return of smallpox. N Engl J Med (2003) 348:463-6. doi: 10.1056/NEJMp025175

9. Kennedy RB, Ovsyannikova I, Poland GA. Smallpox vaccines for biodefense. Vaccine (2009) 27(Suppl 4):D73-9. doi: 10.1016/j.vaccine.2009.07.103

10. Kennedy RB, Ovsyannikova IG, Jacobson RM, Poland GA. The immunology of smallpox vaccines. Curr Opin Immunol (2009) 21(3):314-20. doi: 10.1016/ j.coi.2009.04.004

11. Kennedy RB, Ovsyannikova IG, Pankratz VS, Haralambieva IH, Vierkant RA, Jacobson RM, et al. Genome-wide genetic associations with IFNgamma response to smallpox vaccine. Hum Gen (2012) 131(9):1433-51. doi: 10.1007/s00439-012-1179-x

12. Ovsyannikova IG, Kennedy RB, O’Byrne M, Jacobson RM, Pankratz VS, Poland GA. Genome-wide association study of antibody response to smallpox vaccine. Vaccine (2012) 30(28):4182-9. doi: 10.1016/j.vaccine.2012.04.055

13. Kennedy RB, Poland GA, Ovsyannikova IG, Oberg AL, Asmann YW, Grill $\mathrm{DE}$, et al. Impaired innate, humoral, and cellular immunity despite a take in smallpox vaccine recipients. Vaccine (2016) 34(28):3283-90. doi: 10.1016/ j.vaccine.2016.05.005 
14. Crowe JEJr. Genetic predisposition for adverse events after vaccination. J Infect Dis (2007) 196(2):176-7. doi: 10.1086/518800

15. Kennedy RB, Ovsyannikova IG, Shane PV, Haralambieva IH, Vierkant RA, Poland GA. Genome-wide analysis of polymorphisms associated with cytokine responses in smallpox vaccine recipients. Hum Gen (2012) 131 (9):1403-21. doi: 10.1007/s00439-012-1174-2

16. Motwani M, Pesiridis S, Fitzgerald KA. DNA sensing by the cGAS-STING pathway in health and disease. Nat Rev Genet (2019) 20(11):657-74. doi: 10.1038/s41576-019-0151-1

17. Tan X, Sun L, Chen J, Chen ZJ. Detection of Microbial Infections Through Innate Immune Sensing of Nucleic Acids. Annu Rev Microbiol (2018) 72:44778. doi: 10.1146/annurev-micro-102215-095605

18. Ferguson BJ, Mansur DS, Peters NE, Ren H, Smith GL. DNA-PK is a DNA sensor for IRF-3-dependent innate immunity. eLife (2012) 1:e00047. doi: 10.7554/eLife.00047

19. Georgana I, Sumner RP, Towers GJ, Maluquer de Motes C. Virulent poxviruses inhibit DNA sensing by preventing STING activation. J Virol (2018) 92(10):e02145-17. doi: 10.1128/JVI.02145-17

20. Unterholzner L, Sumner RP, Baran M, Ren H, Mansur DS, Bourke NM, et al. Vaccinia virus protein C6 is a virulence factor that binds TBK-1 adaptor proteins and inhibits activation of IRF3 and IRF7. PLoS Pathog (2011) 7(9): e1002247. doi: 10.1371/journal.ppat.1002247

21. Cao H, Dai P, Wang W, Li H, Yuan J, Wang F, et al. Innate immune response of human plasmacytoid dendritic cells to poxvirus infection is subverted by vaccinia E3 via its Z-DNA/RNA binding domain. PLoS One (2012) 7(5): e36823. doi: 10.1371/journal.pone.0036823

22. Yi G, Brendel VP, Shu C, Li P, Palanathan S, Cheng Kao C. Single nucleotide polymorphisms of human STING can affect innate immune response to cyclic dinucleotides. PLoS One (2013) 8(10):e77846. doi: 10.1371/journal.pone.0077846

23. Tsung K, Yim JH, Marti W, Buller RM, Norton JA. Gene expression and cytopathic effect of vaccinia virus inactivated by psoralen and long-wave UV light. J Virol (1996) 70(1):165-71. doi: 10.1128/JVI.70.1.165-171.1996

24. Ovsyannikova IG, Pankrantz VS, Salk HM, Kennedy RB, Poland GA. HLA alleles associated with the adaptive immune response to smallpox vaccine: a replication study. Hum Genet (2014) 133(9):1083-92. doi: 10.1007/s00439014-1449-x

25. Haralambieva IH, Ovsyannikova IG, Kennedy RB, Larrabee BR, Shane Pankratz V, Poland GA. Race and sex-based differences in cytokine immune responses to smallpox vaccine in healthy individuals. Hum Immunol (2013) 74(10):1263-6. doi: 10.1016/j.humimm.2013.06.031

26. Delaneau O, Howie B, Cox AJ, Zagury JF, Marchini J. Haplotype estimation using sequencing reads. Am J Hum Genet (2013) 93(4):687-96. doi: 10.1016/ j.ajhg.2013.09.002

27. Howie BN, Donnelly P, Marchini J. A flexible and accurate genotype imputation method for the next generation of genome-wide association studies. PLoS Genet (2009) 5(6):e1000529. doi: 10.1371/journal.pgen.1000529

28. Pritchard JK, Stephens M, Donnelly P. Inference of population structure using multilocus genotype data. Genetics (2000) 155(2):945-59.

29. Ovsyannikova IG, Schaid DJ, Larrabee BR, Haralambieva IH, Kennedy RB, Poland GA. A large population-based association study between HLA and KIR genotypes and measles vaccine antibody responses. PLoS One (2017) 12 (2):e0171261. doi: 10.1371/journal.pone.0171261

30. Novembre J, Stephens M. Interpreting principal component analyses of spatial population genetic variation. Nat Genet (2008) 40(5):646-9. doi: 10.1038/ng.139

31. Price AL, Patterson NJ, Plenge RM, Weinblatt ME, Shadick NA, Reich D. Principal components analysis corrects for stratification in genome-wide association studies. Nat Genet (2006) 38(8):904-9. doi: 10.1038/ng1847

32. Manolio TA. Genomewide association studies and assessment of the risk of disease. N Engl J Med (2010) 363(2):166-76. doi: 10.1056/NEJMra0905980

33. Pe'er I, Yelensky R, Altshuler D, Daly MJ. Estimation of the multiple testing burden for genomewide association studies of nearly all common variants. Genet Epidemiol (2008) 32(4):381-5. doi: 10.1002/gepi.20303

34. Purcell S, Neale B, Todd-Brown K, Thomas L, Ferreira MA, Bender D, et al. PLINK: a tool set for whole-genome association and population-based linkage analyses. Am J Hum Genet (2007) 81(3):559-75. doi: 10.1086/519795

35. Voigt EA, Yin J. Kinetic Differences and Synergistic Antiviral Effects Between Type I and Type III Interferon Signaling Indicate Pathway Independence. J Interf Cytokine Res (2015) 35(9):734-47. doi: 10.1089/jir.2015.0008
36. Antoniak S, Owens AP,3, Baunacke M, Williams JC, Lee RD, Weithauser A, et al. PAR- 1 contributes to the innate immune response during viral infection. J Clin Invest (2013) 123(3):1310-22. doi: 10.1172/JCI66125

37. Huang YH, Liu XY, Du XX, Jiang ZF, Su XD. The structural basis for the sensing and binding of cyclic di-GMP by STING. Nat Struct Molec Biol (2012) 19(7):728-30. doi: 10.1038/nsmb.2333

38. Biasini M, Bienert S, Waterhouse A, Arnold K, Studer G, Schmidt T, et al. SWISS-MODEL: modelling protein tertiary and quaternary structure using evolutionary information. Nucleic Acids Res (2014) 42(Web Server issue): W252-8. doi: 10.1093/nar/gku340

39. Gao P, Zillinger T, Wang W, Ascano M, Dai P, Hartmann G, et al. Bindingpocket and lid-region substitutions render human STING sensitive to the species-specific drug DMXAA. Cell Rep (2014) 8(6):1668-76. doi: 10.1016/ j.celrep.2014.08.010

40. Shang G, Zhu D, Li N, Zhang J, Zhu C, Lu D, et al. Crystal structures of STING protein reveal basis for recognition of cyclic di-GMP. Nat Struct Molec Biol (2012) 19(7):725-7. doi: 10.1038/nsmb.2332

41. Phillips JC, Braun R, Wang W, Gumbart J, Tajkhorshid E, Villa E, et al. Scalable molecular dynamics with NAMD. J Comput Chem (2005) 26 (16):1781-802. doi: $10.1002 /$ jcc.20289

42. Mackerell ADJr., Feig M, Brooks CL3rd. Extending the treatment of backbone energetics in protein force fields: limitations of gas-phase quantum mechanics in reproducing protein conformational distributions in molecular dynamics simulations. J Comput Chem (2004) 25(11):1400-15. doi: 10.1002/jcc.20065

43. Sethi S, Dasari S, Plaisier E, Ronco P, Nasr SH, Brocheriou I, et al. Apolipoprotein CII Amyloidosis Associated With p.Lys41Thr Mutation. Kidney Int Rep (2018) 3(5):1193-201. doi: 10.1016/j.ekir.2018.04.009

44. Rashin AA, Domagalski MJ, Zimmermann MT, Minor W, Chruszcz M, Jernigan RL. Factors correlating with significant differences between X-ray structures of myoglobin. Acta Crystallogr D Biol Crystallogr (2014) 70(Pt 2):481-91. doi: 10.1107/S1399004713028812

45. Holm L, Sander C. Protein structure comparison by alignment of distance matrices. J Molec Biol (1993) 233(1):123-38. doi: 10.1006/jmbi.1993.1489

46. Grant BJ, Rodrigues AP, ElSawy KM, McCammon JA, Caves LS. Bio3d: an R package for the comparative analysis of protein structures. Bioinformatics (2006) 22(21):2695-6. doi: 10.1093/bioinformatics/btl461

47. Humphrey W, Dalke A, Schulten K. VMD: visual molecular dynamics. J Molec Graph (1996) 14(1):33-8, 27-8. doi: 10.1016/0263-7855(96)00018-5

48. Umlauf BJ, Ovsyannikova IG, Haralambieva IH, Kennedy RB, Vierkant RA, Pankratz VS, et al. Correlations between vaccinia-specific immune responses within a cohort of armed forces members. Viral Immunol (2011) 24(5):41520. doi: 10.1089 /vim.2011.0029

49. Dai P, Wang W, Cao H, Avogadri F, Dai L, Drexler I, et al. Modified vaccinia virus Ankara triggers type I IFN production in murine conventional dendritic cells via a cGAS/STING-mediated cytosolic DNA-sensing pathway. PLoS Pathog (2014) 10(4):e1003989. doi: 10.1371/journal.ppat.1003989

50. Jin L, Xu LG, Yang IV, Davidson EJ, Schwartz DA, Wurfel MM, et al. Identification and characterization of a loss-of-function human MPYS variant. Genes Immun (2011) 12(4):263-9. doi: 10.1038/gene.2010.75

51. Schaid DJ, Rowland CM, Tines DE, Jacobson RM, Poland GA. Score tests for association between traits and haplotypes when linkage phase is ambiguous. Am J Hum Genet (2002) 70:425-34. doi: 10.1086/338688

52. Sun L, Wu J, Du F, Chen X, Chen ZJ. Cyclic GMP-AMP synthase is a cytosolic DNA sensor that activates the type I interferon pathway. Science (2013) 339 (6121):786-91. doi: 10.1126/science.1232458

53. Quigley M, Martinez J, Huang X, Yang Y. A critical role for direct TLR2MyD88 signaling in CD8 T-cell clonal expansion and memory formation following vaccinia viral infection. Blood (2009) 113(10):2256-64. doi: 10.1182/ blood-2008-03-148809

54. Zhao Y, De Trez C, Flynn R, Ware CF, Croft M, Salek-Ardakani S. The adaptor molecule MyD88 directly promotes CD8 T cell responses to vaccinia virus. J Immunol (2009) 182(10):6278-86. doi: 10.4049/jimmunol.0803682

55. Zhang X, Shi H, Wu J, Sun L, Chen C, Chen ZJ. Cyclic GMP-AMP containing mixed phosphodiester linkages is an endogenous high-affinity ligand for STING. Molecr Cell (2013) 51(2):226-35. doi: 10.1016/j.molcel.2013.05.022

56. Patel S, Blaauboer SM, Tucker HR, Mansouri S, Ruiz-Moreno JS, Hamann L, et al. Correction: The Common R71H-G230A-R293Q Human TMEM173 Is a Null Allele. J Immunol (2017) 198(11):4547. doi: 10.4049/jimmunol.1700477 
57. Patel S, Blaauboer SM, Tucker HR, Mansouri S, Ruiz-Moreno JS, Hamann L, et al. The Common R71H-G230A-R293Q Human TMEM173 Is a Null Allele. J Immunol (2017) 198(2):776-87. doi: 10.4049/jimmunol.1601585

58. Gao P, Ascano M, Zillinger T, Wang W, Dai P, Serganov AA, et al. Structurefunction analysis of STING activation by $c\left[G\left(2^{\prime}, 5^{\prime}\right) \mathrm{pA}\left(3^{\prime}, 5^{\prime}\right) \mathrm{p}\right]$ and targeting by antiviral DMXAA. Cell (2013) 154(4):748-62. doi: 10.1016/j.cell. 2013.07.023

Conflict of Interest: GP is the chair of a Safety Evaluation Committee for novel investigational vaccine trials being conducted by Merck Research Laboratories. GP offers consultative advice on vaccine development to Merck \& Co., Medicago, GlaxoSmithKline, Sanofi Pasteur, Emergent Biosolutions, Dynavax, Genentech, Eli Lilly and Company, Janssen Global Services LLC, Kentucky Bioprocessing, and Genevant Sciences, Inc. GP, RK, and IO hold patents related to vaccinia, influenza, and measles peptide vaccines. GP, RK, and IO have received grant funding from ICW Ventures for preclinical studies on a peptide-based COVID-19 vaccine. RK has received funding from Merck Research Laboratories to study waning immunity to mumps vaccine. These activities and this research have been reviewed by the Mayo Clinic Conflict of Interest Review Board and are conducted in compliance with Mayo Clinic Conflict of Interest policies.

The remaining authors declare that the research was conducted in the absence of any commercial or financial relationships that could be construed as a potential conflict of interest.

Copyright (๑) 2020 Kennedy, Haralambieva, Ovsyannikova, Voigt, Larrabee, Schaid, Zimmermann, Oberg and Poland. This is an open-access article distributed under the terms of the Creative Commons Attribution License (CC BY). The use, distribution or reproduction in other forums is permitted, provided the original author(s) and the copyright owner(s) are credited and that the original publication in this journal is cited, in accordance with accepted academic practice. No use, distribution or reproduction is permitted which does not comply with these terms. 